\title{
Soft phonon modes driven huge difference on lattice thermal conductivity between topological semimetal WC and WN
}

\author{
San-Dong Guo ${ }^{1}$ and Peng Chen ${ }^{2,3}$ \\ ${ }^{1}$ School of Physics, China University of Mining and Technology, Xuzhou 221116, Jiangsu, China \\ ${ }^{2}$ Beijing National Laboratory for Condensed Matter Physics, \\ Institute of Physics, Chinese Academy of Sciences, Beijing 100190, China and \\ ${ }^{3}$ School of Physical Sciences, University of Chinese Academy of Sciences, Beijing 100190, China
}

\begin{abstract}
Topological semimetals are currently attracting increasing interest due to their potential applications in topological qubits and low-power electronics, which are closely related to their thermal transport properties. In this work, by solving the Boltzmann transport equation based on firstprinciples calculations, we systematically investigate the phonon transport properties of topological semimetal WC and WN. The predicted room-temperature lattice thermal conductivities of WC (WN) along a and c directions are $1140.64(7.47) \mathrm{Wm}^{-1} \mathrm{~K}^{-1}$ and 1214.69 (5.39) $\mathrm{Wm}^{-1} \mathrm{~K}^{-1}$. Considering the similar crystal structure of $\mathrm{WC}$ and $\mathrm{WN}$, it is quite interesting to find that the thermal conductivity of WC is more than two orders of magnitude higher than that of WN. It is found that, different from WN, the large acoustic-optical (a-o) gap prohibits the acoustic+acoustic $\rightarrow$ optical (aao) scattering, which gives rise to very long phonon lifetimes, leading to ultrahigh lattice thermal conductivity in WC. For WN, the lack of a-o gap is due to soft phonon modes in optical branches, which can provide more scattering channels for aao scattering, producing very short phonon lifetimes. Further deep insight can be attained from their different electronic structures. Distinctly different from that in WC, the density of states (DOS) of WN at the Fermi level becomes very sharp, which leads to destabilization of WN, producing soft phonon modes. It is found that the small shear modulus $G$ and $C_{44}$ limit the stability of WN, compared with WC. Our works provide valuable information for phonon transports in $\mathrm{WC}$ and $\mathrm{WN}$, and motivate further experimental works to study their lattice thermal conductivities.
\end{abstract}

PACS numbers: 72.15.Jf, 71.20.-b, 71.70.Ej, 79.10.-n

Keywords: Lattice thermal conductivity; Group velocities; Phonon lifetimes

Email:guosd@cumt.edu.cn

\section{INTRODUCTION}

Topological semimetals have attracted great research interest in condensed matter physics and material science $^{1-6}$. In three dimensions, the band crossing of topological semimetals may occur at discrete points (Weyl/Dirac semimetals) ${ }^{1,2}$, or along closed loops (topological nodal line semimetals) ${ }^{5,7}$. The $\mathrm{Na}_{3} \mathrm{Bi}$ (Dirac semimetal) and TaAs (Weyl semimetal) have been confirmed by angle-resolved photoemission spectroscopy (ARPES) $)^{1,3,8-10}$. Besides Dirac/Weyl fermion (four/twofold degenerate point), three-, six- or eight-fold band crossings have been proposed as new types of topological semimetals ${ }^{11}$. The three-fold degenerate crossing points have been predicted in a family of two-element metals $\mathrm{AB}(\mathrm{A}=\mathrm{Zr}, \mathrm{Nb}, \mathrm{Mo}, \mathrm{Ta}, \mathrm{W} ; \mathrm{B}=\mathrm{C}, \mathrm{N}, \mathrm{P}, \mathrm{S}, \mathrm{Te})$ with WC-type structure ${ }^{12-14}$, MoP of which has been experimentally confirmed with triply degenerate nodal points (TDNPs), coexisting with the pairs of Weyl points ${ }^{4}$.

Topological semimetal may have substantial applications in topological qubits, spintronics and quantum computations ${ }^{15}$. Efficient heat dissipation can ensure the reliability and stability of topological semimetalbased electronics devices. This is closely related to thermal transport, and high lattice thermal conductivity is beneficial to efficient heat dissipation ${ }^{16}$. The lattice thermal conductivities of topological semimetals TaAs, MoP, ZrTe and TaN have been predicted by first-

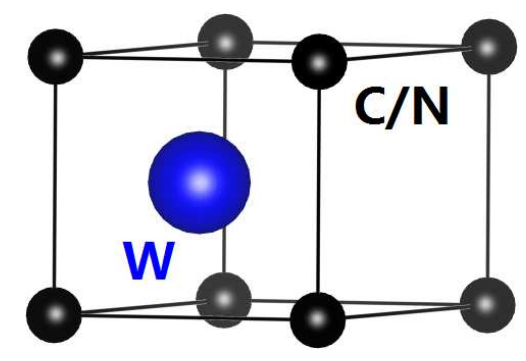

FIG. 1. (Color online)The crystal structure of $\mathrm{WC} / \mathrm{N}$ in one unit cell, and the blue and black balls represent $\mathrm{W}$ and $\mathrm{C} / \mathrm{N}$ atoms, respectively.

principles calculations and the linearized phonon Boltzmann equation ${ }^{17-21}$. The lattice thermal conductivities of TaAs, MoP and ZrTe are relatively low, and the roomtemperature ones are about $17 \sim 44 \mathrm{Wm}^{-1} \mathrm{~K}^{-117-20}$. However, the room-temperature lattice thermal conductivity of TaN is ultrahigh, $838.62 \mathrm{Wm}^{-1} \mathrm{~K}^{-1}$ along a axis and $1080.40 \mathrm{Wm}^{-1} \mathrm{~K}^{-1}$ along $\mathrm{c}$ axis, which is due to very large a-o gap ${ }^{21}$. In two-element metals $\mathrm{AB}$, much different atomic masses of $\mathrm{A}$ and $\mathrm{B}$ atoms may lead to large a-o gap. Based on mass difference factor, the WC and WN may have ultrahigh lattice thermal conductivities $^{21}$, which needs to be further confirmed by the first-principles calculations.

In this work, the phonon transport properties of WC and WN are studied by solving the phonon Boltzmann 


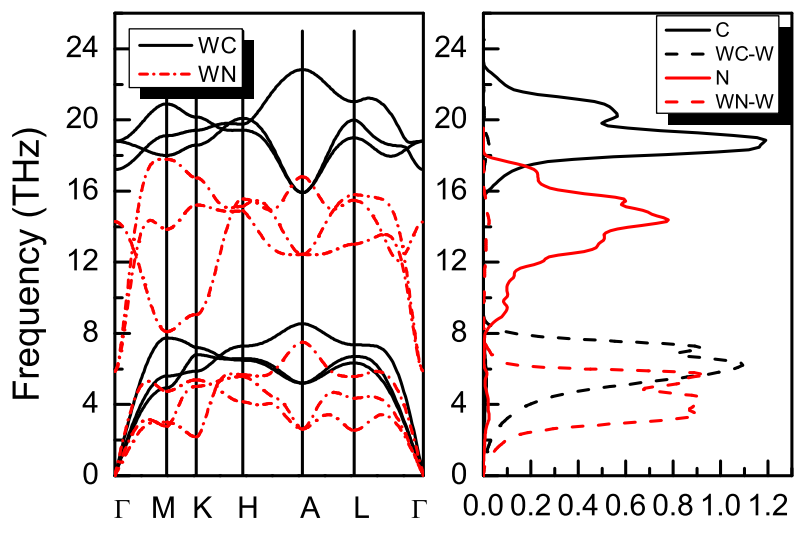

FIG. 2. (Color online) Phonon dispersion curves of WC and WN with the corresponding atom partial DOS.

transport equation. It is found that the thermal conductivity of WC is more than two orders of magnitude higher than that of WN. This can be attributed to the very large a-o gap for WC, and the lack of a-o gap for WN due to soft phonon modes in optical branches. The soft phonon modes can be explained by DOS of WN at the Fermi level. Different from that in WC, the DOS of WN at the Fermi level becomes very sharp, which induces destabilization of WN, leading to soft phonon modes. Compared with WC and TaN, the small shear modulus $G$ and $C_{44}$ may limit the stability of WN.

The rest of the paper is organized as follows. In the next section, we shall give our computational details about phonon transport. In the third section, we shall present phonon transports of WC and WN. Finally, we shall give our discussions and conclusions in the fourth section.

\section{COMPUTATIONAL DETAIL}

We perform the first-principles calculations within the projected augmented wave (PAW) method, as implemented in the VASP code $^{22,23,25}$. With the plane-wavecut-off energy of $500 \mathrm{eV}$, the generalized gradient approximation of the Perdew-Burke-Ernzerhof (GGA-PBE) ${ }^{24}$ is used for the exchange-correlation functional. The $6 \mathrm{~s}$ and $5 \mathrm{~d}$ electrons of $\mathrm{W}$, and $2 \mathrm{~s}$ and $2 \mathrm{p}$ electrons of $\mathrm{C} / \mathrm{N}$ are treated as valance electrons. The convergence criteria is with energy convergences being less than $10^{-8} \mathrm{eV}$. The lattice thermal conductivities of WC and WN are predicted by solving linearized phonon Boltzmann equation with the single-mode relaxation time approximation (RTA), as implemented in the Phono3py code ${ }^{26}$. The lattice thermal conductivity can be attained by the following formula:

$$
\kappa=\frac{1}{N V_{0}} \sum_{\lambda} \kappa_{\lambda}=\frac{1}{N V_{0}} \sum_{\lambda} C_{\lambda} \nu_{\lambda} \otimes \nu_{\lambda} \tau_{\lambda}
$$

where $\lambda$ is phonon mode, $N$ is the total number of $\mathrm{q}$ points sampling the Brillouin zone (BZ), $V_{0}$ is the volume of a unit cell, and $C_{\lambda}, \nu_{\lambda}, \tau_{\lambda}$ is the specific heat, phonon velocity, phonon lifetime. The phonon lifetime $\tau_{\lambda}$ can be calculated by phonon linewidth $2 \Gamma_{\lambda}\left(\omega_{\lambda}\right)$ of the phonon mode $\lambda$ :

$$
\tau_{\lambda}=\frac{1}{2 \Gamma_{\lambda}\left(\omega_{\lambda}\right)}
$$

The $\Gamma_{\lambda}(\omega)$ takes the form analogous to the Fermi golden rule:

$$
\begin{gathered}
\Gamma_{\lambda}(\omega)=\frac{18 \pi}{\hbar^{2}} \sum_{\lambda^{\prime} \lambda^{\prime \prime}}\left|\Phi_{-\lambda \lambda^{\prime} \lambda^{\prime \prime}}\right|^{2}\left[( f _ { \lambda } ^ { \prime } + f _ { \lambda } ^ { \prime \prime } + 1 ) \delta \left(\omega-\omega_{\lambda}^{\prime}-\right.\right. \\
\left.\left.\omega_{\lambda}^{\prime \prime}\right)+\left(f_{\lambda}^{\prime}-f_{\lambda}^{\prime \prime}\right)\left[\delta\left(\omega+\omega_{\lambda}^{\prime}-\omega_{\lambda}^{\prime \prime}\right)-\delta\left(\omega-\omega_{\lambda}^{\prime}+\omega_{\lambda}^{\prime \prime}\right)\right]\right]
\end{gathered}
$$

in which $f_{\lambda}$ is the phonon equilibrium occupancy and $\Phi_{-\lambda \lambda^{\prime} \lambda^{\prime \prime}}$ is the strength of interaction among the three phonons $\lambda, \lambda^{\prime}$, and $\lambda^{\prime \prime}$ involved in the scattering.

Based on the supercell approach with finite atomic displacement of $0.03 \AA$, the second- and third-order interatomic force constants (IFCs) can be attained. For second-order harmonic IFCs, a $4 \times 4 \times 4$ supercell containing 128 atoms is used with k-point meshes of $2 \times$ $2 \times 2$. According to the harmonic IFCs, phonon dispersions of WC and WN can be attained by Phonopy package $^{27}$, determining the allowed three-phonon scattering processes. The group velocity and specific heat can also be compulated from phonon dispersion. For the third-order anharmonic IFCs, a $3 \times 3 \times 3$ supercells containing 54 atoms is used with k-point meshes of $3 \times 3$ $\times 3$. The three-phonon scattering rate can be attained by third-order anharmonic IFCs, and then the phonon lifetimes can be calculated. To compute lattice thermal conductivities, the reciprocal spaces of the primitive cells are sampled using the $20 \times 20 \times 20$ meshes.

\section{MAIN CALCULATED RESULTS AND ANALYSIS}

The low-temperature WC and WN have a hexagonal structure with space group $P \overline{6} m 2$ (No. 187), where W and $\mathrm{C}(\mathrm{N})$ atoms occupy the $1 \mathrm{~d}(1 / 3,2 / 3,1 / 2)$ and 1a $(0,0,0)$ Wyckoff positions, respectively. The crystal structure is shown in Figure 1. The experimental lattice constants of WC $(a=b=2.928 \AA, c=2.835 \AA)$ and WN $(a=b=2.890 \AA, c=2.830 \AA)^{14}$ are used to investigate their phonon transport properties. The phonon dispersions of WC and WN along several high symmetry paths are plotted in Figure 2, along with atom partial DOS. The 3 acoustic and 3 optical phonon branches are observed due to two atoms in one unit cell. There are no imaginary frequencies in the phonon dispersions of both $\mathrm{WC}$ and $\mathrm{WN}$, which indicates the thermodynamic stability of WC and WN with hexagonal structure. A very 
TABLE I. The elastic constants $\left(C_{11}, C_{12}, C_{13}, C_{33}, C_{44}\right.$ and $\left.C_{66}\right)$, bulk $(B)$, shear $(G)$ moduli (in GPa) and $B / G$ of WC, WN and TaN.

\begin{tabular}{cccccccccc}
\hline \hline Name & $C_{11}$ & $C_{12}$ & $C_{13}$ & $C_{33}$ & $C_{44}$ & $C_{66}$ & $B$ & $G$ & $B / G$ \\
\hline \hline WC & 709.58 & 194.81 & 171.92 & 954.54 & 300.76 & 257.38 & 380.87 & 291.72 & 1.31 \\
\hline WN & 637.37 & 200.34 & 283.65 & 756.06 & 77.06 & 218.52 & 392.50 & 141.65 & 2.77 \\
\hline TaN & 566.40 & 128.23 & 62.41 & 706.39 & 215.02 & 219.09 & 260.26 & 233.16 & 1.12 \\
\hline \hline
\end{tabular}
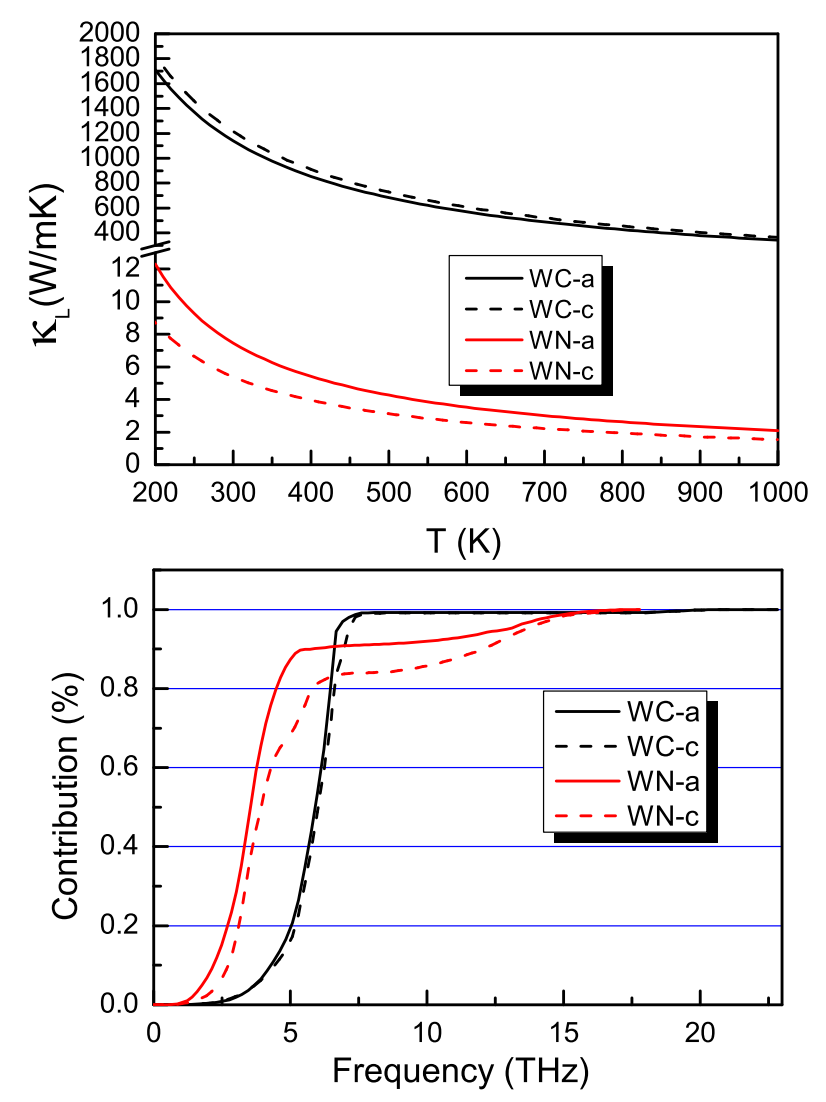

FIG. 3. (Color online) The lattice thermal conductivities of $\mathrm{WC}$ and $\mathrm{WN}$ as a function of temperature, including a and $\mathrm{c}$ directions; the cumulative lattice thermal conductivities (300 $\mathrm{K}$ ) of WC and WN divided by total lattice thermal conductivity with respect to phonon frequency, along a and c directions.

wide a-o gap of $7.37 \mathrm{THz}$ is observed for WC, which is very close to width of acoustic branches $(8.55 \mathrm{THz})$. The large a-o gap is due to very different atomic masses of $\mathrm{W}$ and $\mathrm{C}$ atoms ${ }^{28,29}$. Similar results can be found in topological semimetal $\mathrm{TaN}^{21}$. Compared to WC, the optical branches of WN exist soft phonon modes near $\Gamma$, $\mathrm{M}$ and $\mathrm{K}$ points. Then, the acoustic and optical modes of WN are not separated by the large band gap, despite very different atomic masses for $\mathrm{W}$ and $\mathrm{N}$ atoms. The size of a-o gap can produce very important effects on aao scattering ${ }^{30,31}$. It is found that acoustic (optical) branches of $\mathrm{WC} / \mathrm{N}$ are mainly contributed by the vibrations of $\mathrm{W}(\mathrm{C} / \mathrm{N})$ atoms from atom partial DOS.

The lattice thermal conductivities of $\mathrm{WC}$ and $\mathrm{WN}$
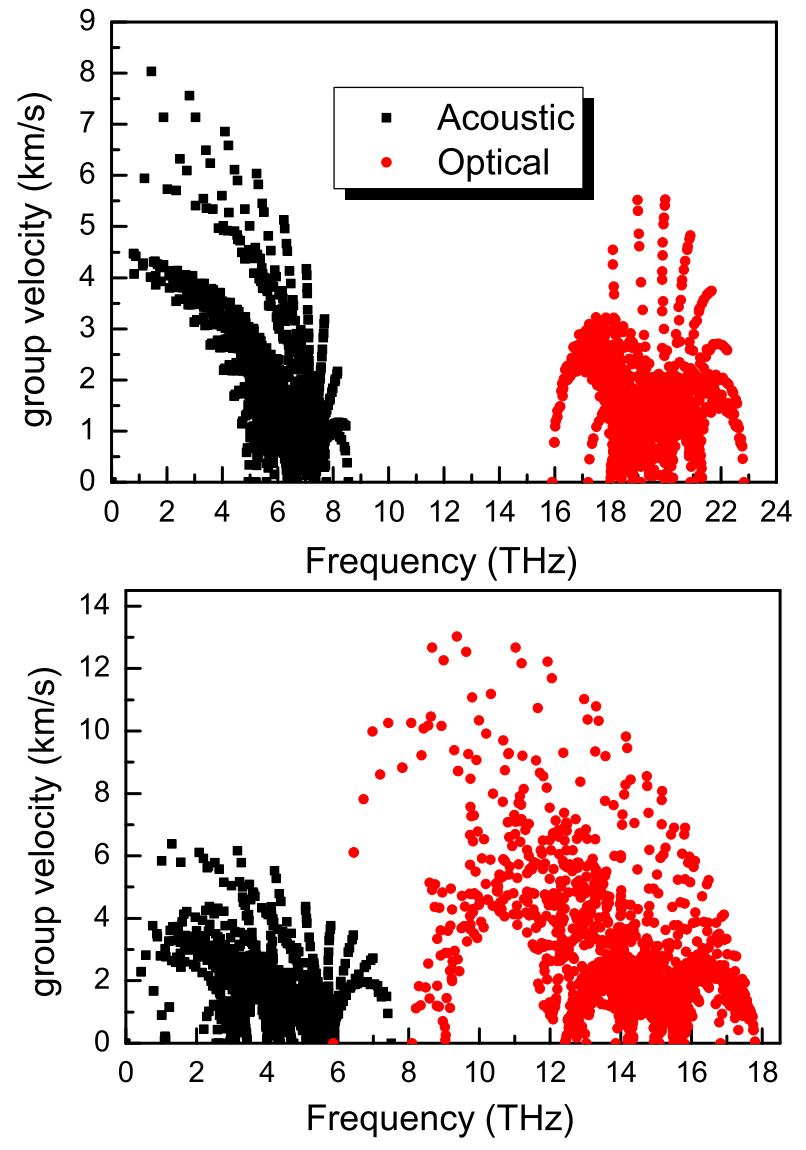

FIG. 4. (Color online) The mode level phonon group velocities of WC (Top) and WN (Bottom) in the first Brillouin zone.

along a and c directions as a function of temperature are shown in Figure 3. Due to intrinsic enhancement of phonon-phonon scattering, the lattice thermal conductivities decrease with increasing temperature in the considered temperature region. The lattice thermal conductivity of WC along $\mathrm{c}$ direction is higher than that along a direction, which is consistent with that of TaAs, ZrTe, MoP and $\mathrm{TaN}^{17-21}$. But it is opposite for WN. It is clearly seen that the lattice thermal conductivity of $\mathrm{WC}$ is hundreds of times higher than one of WN in the considered temperature region. The room-temperature lattice thermal conductivities of WC along a and c directions are $1140.64 \mathrm{Wm}^{-1} \mathrm{~K}^{-1}$ and $1214.69 \mathrm{Wm}^{-1} \mathrm{~K}^{-1}$, and $7.47 \mathrm{Wm}^{-1} \mathrm{~K}^{-1}$ and $5.39 \mathrm{Wm}^{-1} \mathrm{~K}^{-1}$ for $\mathrm{WN}$. The lattice thermal conductivity of $\mathrm{WC}$ is close to that of 

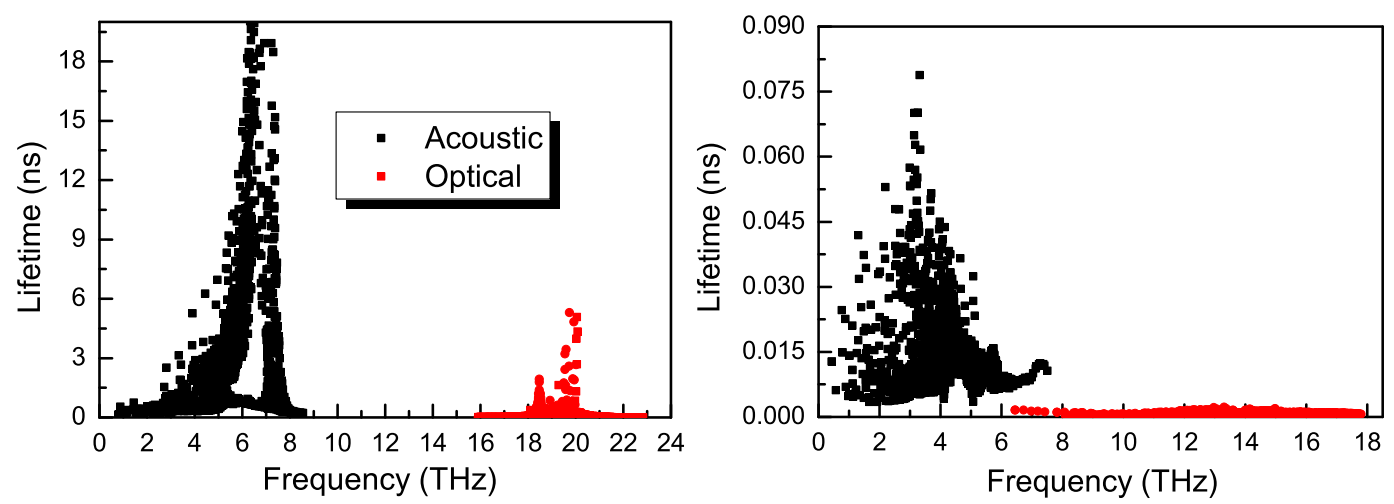

FIG. 5. (Color online) The mode level phonon lifetimes (300K) of WC (Left) and WN (Right) in the first Brillouin zone.

$\mathrm{TaN}^{21}$, which is very higher than that of TaAs, ZrTe and $\mathrm{MoP}^{17-20}$. However, the one of WN is lower than that of TaAs, ZrTe and MoP. An anisotropy factor ${ }^{19}$ $\left(\eta=\left(\kappa_{L}(\max )-\kappa_{L}(\min )\right) / \kappa_{L}(\min )\right)$ is defined to measure the anisotropic strength of lattice thermal conductivities along a and $\mathrm{c}$ directions, and the corresponding value is $6.5 \%$ for $\mathrm{WC}$ and $38.6 \%$ for $\mathrm{WN}$, implying weaker anisotropy for WC than WN. At room temperature, the cumulative lattice thermal conductivities divided by total lattice thermal conductivity (CLDT) with respect to frequency along a and c directions are plotted in Figure 3. It is clearly seen that acoustic branches dominate lattice thermal conductivity for both WC and WN. For WC, the CLDTs along a and c directions almost coincide, showing weak anisotropy. The CLDTs of WN along a and c directions show significant difference, which agrees well with strong anisotropy of lattice thermal conductivity. It is found that optical branches of WN have obvious contribution to total lattice thermal conductivity.

To gain more insight into huge difference on lattice thermal conductivity between WC and WN, the mode level phonon group velocities and lifetimes are shown in Figure 4 and Figure 5, respectively. The most of group velocities of acoustic branches for $\mathrm{WC}$ are larger than those of WN, while it is opposite for the optical branches. Due to dominated contribution to total lattice thermal conductivity from acoustic branches, group velocities can partially explain higher lattice thermal conductivity for WC than WN. Because of group velocities in the same order of magnitude between $\mathrm{WC}$ and $\mathrm{WN}$, it can not account for huge difference on lattice thermal conductivity. It is found that the most of group velocities of WC for acoustic branches are higher than those of optical branches, but it is opposite for WN. From Figure 5, it is clearly seen that phonon lifetimes of WC are hundreds of times longer than ones of WN, which leads to extremely higher lattice thermal conductivity for WC than WN. Such long phonon lifetimes can also be found in $\mathrm{TaN}^{21}$. Although acoustic branches dominate heat transport, the optical branches can provide scattering channels for the acoustic modes to achieve three phonon scattering, especially for aao scattering. For WC, the huge a-o gap
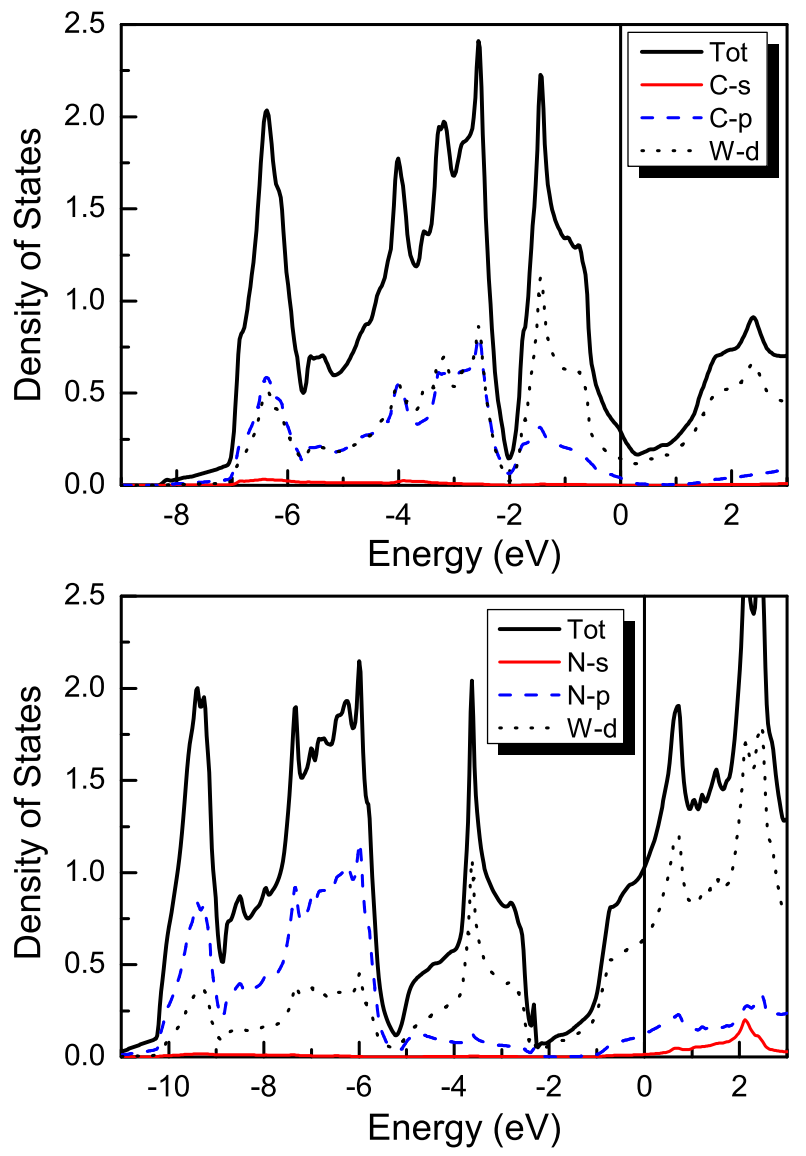

FIG. 6. (Color online) The total and projected DOSs of WC (Top) and WN (Bottom).

impedes the annihilation process of two acoustic phonon modes into one optical one due to the requirement on energy conservation, which leads to weak phonon-phonon scattering rate, resulting in very long phonon lifetimes. Similar mechanism can also be found in $\mathrm{TaN}^{21}, \mathrm{BAs}^{30}$ and $\mathrm{AlSb}^{31}$. For WN, the aao scattering can be easily achieved due to absent a-o gap, which gives to very short phonon lifetimes. 

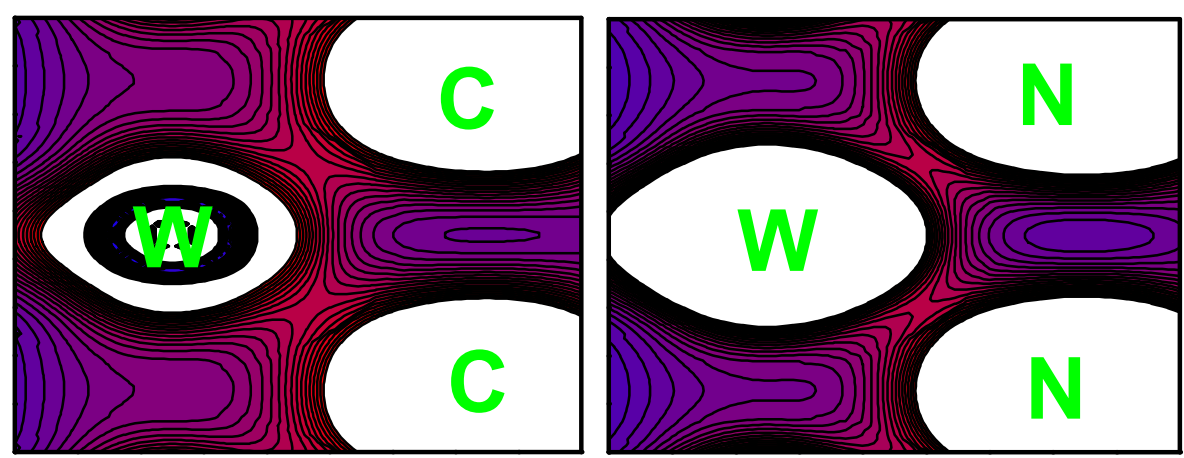

FIG. 7. (Color online) The charge density distributions of WC (left) and WN (Right) [unit:|e|/bohr ${ }^{3}$ in the (110) plane. The charge density value increases from blue to red.

In spite of very different atomic masses for $\mathrm{W}$ and $\mathrm{N}$ atoms, the a-o gap of $\mathrm{WN}$ disappears, which is due to soft phonon modes in optical branches. The soft phonon modes mean that WN has weaker stability than WC. In order to get a deep insight into the very different nature between WC and WN, we further perform analysis from the view of electronic structures. The atomic structure and behavior of electrons fundamentally determine all the properties of a material ${ }^{32}$. The total and partial DOSs of $\mathrm{WC}$ and $\mathrm{WN}$ are plotted in Figure 6. For WC, the strongly hybridized $\mathrm{W}-5 \mathrm{~d}$ and $\mathrm{C}-2 \mathrm{p}$ states are observed from $-7.0 \mathrm{eV}$ to $-2.0 \mathrm{eV}$, which is indicative of strong covalent bonding. However, a weak ionic component is observed due to a partial charge transfer from $\mathrm{W}$ to $\mathrm{C}$ atoms from Figure 7. The W-5d states have main contribution from $-2.0 \mathrm{eV}$ to Fermi level. The Fermi level is close to the DOS minimum, which qualitatively declares the stability of WC. If the Fermi level lies at the local DOS maximum, this will induce destabilization. A similar hybridization picture is observed for WN, but an additional electron further fills W-5d states compared with WC, where the DOS at the Fermi level increases sharply. This leads to a destabilization of $\mathrm{WN}$, inducing soft phonon modes in optical branches. Compared with C-2p and W-5d states of WC, the hybridization of W-5d and N-2p states of WN decreases due to enhanced energy separation between $\mathrm{N}$ $2 \mathrm{p}$ and $\mathrm{W}-5 \mathrm{~d}$ states. This leads to weaken covalent bonds compared with isostructural WC. The weakness of covalent bonds from WC to WN can also be easily observed from charge density distributions in Figure 7.

The weaker stability for WN than WC can also be understood by their elastic properties. Due to the hexagonal structure for $\mathrm{WC}$ and $\mathrm{WN}$, five independent elastic constants $\left(C_{11}, C_{12}, C_{13}, C_{33}\right.$ and $\left.C_{44}\right)$ are listed in Table I, which are close to previous calculated values ${ }^{33}$. These elastic constants satisfy the well-known Born stability criteria $^{34,35}$ :

$$
\begin{gathered}
C_{44}>0, C_{11}>0 \\
C_{11}>\left|C_{12}\right| \\
\left(C_{11}+2 C_{12}\right) C_{33}>2 C_{13}^{2}
\end{gathered}
$$
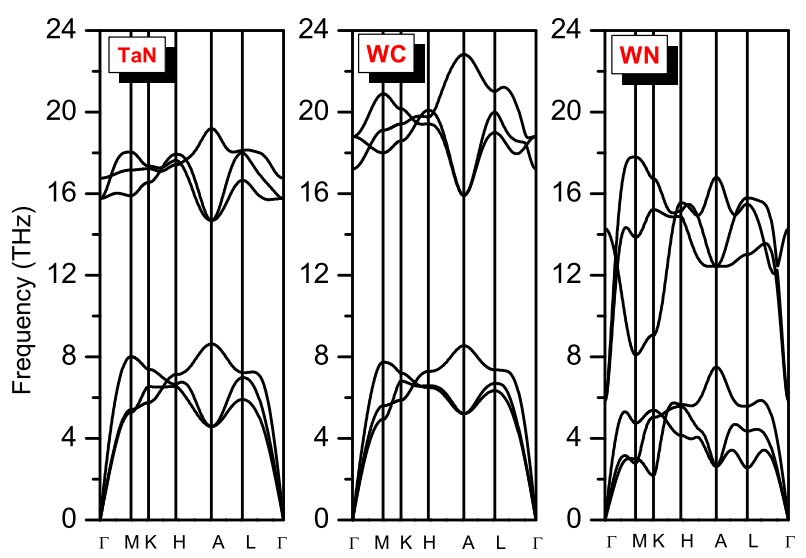

FIG. 8. (Color online)Phonon dispersion curves of TaN, WC and $\mathrm{WN}$.

These mean that WC and WN are mechanically stable. By Voigt-Reuss-Hill approximations, the bulk and shear modulus of WC and WN can be obtained from elastic constants. The Voigt's, Reuss's and Hill's bulk modulus can be calculated by the following equations:

$$
\begin{gathered}
B_{V}=\frac{1}{9}\left(2 C_{11}+C_{33}+2 C_{12}+4 C_{13}\right) \\
B_{R}=\left(2 S_{11}+S_{33}+2 S_{12}+4 S_{13}\right)^{-1} \\
B_{H}=\frac{1}{2}\left(B_{V}+B_{R}\right)
\end{gathered}
$$

The Voigt's, Reuss's and Hill's shear modulus can be attained by using these formulas:

$$
\begin{aligned}
G_{V} & =\frac{1}{15}\left(2 C_{11}+C_{33}-C_{12}-2 C_{13}+6 C_{44}+3 C_{66}\right) \\
G_{R} & =\left[\frac{1}{15}\left(8 S_{11}+4 S_{33}-4 S_{12}-8 S_{13}+6 S_{44}+3 S_{66}\right)\right]^{-1}
\end{aligned}
$$




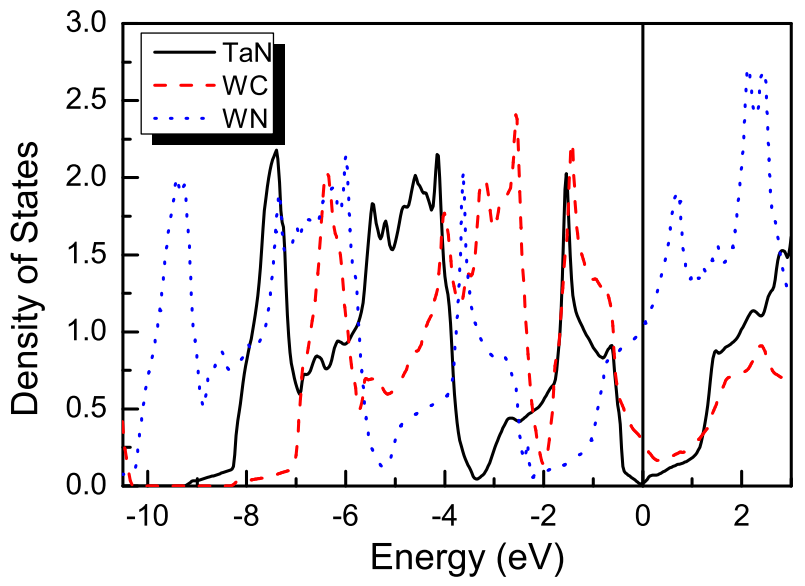

FIG. 9. (Color online) The total DOSs of TaN, WC and WN.

$$
G_{H}=\frac{1}{2}\left(G_{V}+G_{R}\right)
$$

The $S_{i j}$ are the elastic compliance constants. The Hill's bulk and shear modulus are listed in Table I. It is assumed that the hardness of materials can be measured by bulk modulus or shear modulus ${ }^{36}$. For transitionmetal carbonitrides, the magnitude of $C_{44}$ may be a better hardness predictor ${ }^{37,38}$. Therefore, it may be concluded that WC is more harder than WN. The $B / G$ can measure that a material is ductile $(B / G>1.75)$ or brittle $(B / G<1.75)$. Based on $B / G$ in Table I, for WC, the brittle character is dominant. For WN, the increase in the plasticity may be due to enhanced metallicity, caused by high DOS at the Fermi level. It is also found that the shear modulus $G$ and $C_{44}$ may limit the stability of WN.

\section{DISCUSSIONS AND CONCLUSIONS}

The significant mass differences between the constituent atoms can induce a-o gap ${ }^{28,29}$. In some $2 \mathrm{D}$ materials, gap formation mechanism is due to the violation of reflection symmetry selection rule in the harmonic approximation ${ }^{39,40}$. Based on mass difference factor, defined as $\delta=\left(M_{\max }-M_{\min }\right) / M_{\min }, \mathrm{WC}$ and WN may be potential candidates with ultrahigh lattice thermal conductivity due to $\delta$ of $\mathrm{WC}$ and $\mathrm{WN}$ being larger than $10^{21}$. Calculated results show that WC indeed has ultrahigh lattice thermal conductivity, which is the same with TaN with $\delta$ being $11.92^{21}$. However, the WN has very low lattice thermal conductivity. The phonon dispersions of TaN, WC and WN are plotted in Figure 8. The TaN and WC have very similar outlines of phonon dispersion, and a very large a-o gap for TaN $(6.05 \mathrm{THz})$ and $\mathrm{WC}(7.37 \mathrm{THz})$ is observed. The very large a-o gap can produce inefficient aao scattering, which leads to long phonon lifetimes, giving rise to ultrahigh lattice thermal conductivity. A significant difference is observed between $\mathrm{WC} / \mathrm{TaN}$ and $\mathrm{WN}$, and a-o gap of $\mathrm{WN}$ is absent. As a result, the optical branches of WN can provide more scattering channels for the acoustic modes, which leads to strong aao scattering, giving rise to very low lattice thermal conductivity.

Compared with TaN and WC, the lack of a-o gap for WN is due to soft phonon modes in optical branches. The softness of phonon modes can be explained by electronic structures. The DOSs of TaN, WC and WN are plotted in Figure 9. It is clearly seen that the Fermi level for TaN and WC is close to the DOS minimum, while the DOS at the Fermi level for WN increases sharply, which leads to destabilization of WN, inducing soft phonon modes. In fact, they have similar electronic structures. The total number of valence electrons in TaN and WC is ten. Adding one more electron for WN produces softness of the phonon modes due to further filling of W-5d states. Based on elastic properties, it is concluded that the parameter $C_{44}$ and shear modulus $G$ may limit the stability of WN. Based on our calculated results, the mass difference factor and total number of valence electrons in twoelement metals $\mathrm{AB}$ with WC-type structure determine whether they have ultrahigh lattice thermal conductivity.

In summary, we investigate the phonon transport properties of WC and WN by solving the Boltzmann transport equation based on first-principles calculations. Although the WC and WN possess similar crystal structure, they show very diverse lattice thermal conductivity. The room-temperature lattice thermal conductivity for WC is hundreds of times higher than one of WN. The ultrahigh lattice thermal conductivity in $\mathrm{WC}$ is due to the extremely large a-o gap, which strongly restricts aao scattering through the energy conservation, producing very long phonon lifetimes. The very low lattice thermal conductivity in WN is due to softness of phonon modes in optical branches, which can provide more scattering channels for aao scattering, leading to short phonon lifetimes. We further understand the huge difference between WC and WN from the view of electronic structures, considering that all the properties are fundamentally determined by the electronic structures. Distinctly different from that in WC and TaN, the DOS of WN at the Fermi level becomes very sharp, which produces destabilization of WN, leading to soft phonon modes. It is also found that the elastic parameter limiting the stability of WN is the shear modulus $G$ and $C_{44}$. Our works provide fundamental understanding of phonon transport in WC and WN, which will enrich the studies of phonon transport in topological semimetals, and will motivate farther experimental works.

\section{ACKNOWLEDGMENTS}

This work is supported by the National Natural Science Foundation of China (Grant No.11404391). We are grateful to the Advanced Analysis and Computation Center of CUMT for the award of CPU hours to accomplish 
this work.

1 B.Q. Lv, H. M. Weng, B. B. Fu, X. P. Wang, H. Miao, J. Ma, P. Richard, X. C. Huang, L. X. Zhao, G. F. Chen, Z. Fang, X. Dai, T. Qian, and H. Ding, Phys. Rev. X 5, 031013 (2015).

2 Z. J. Wang, Y. Sun, X. Q. Chen, C. Franchini, G. Xu, H. M. Weng, X. Dai, and Z. Fang, Phys. Rev. B 85, 195320 (2012)

3 Z. K. Liu, B. Zhou and Y. Zhang et al., Science 343, 864 (2014).

4 B. Q. Lv, Z. L. Feng, Q.-N. Xu et al., Nature 546, 627 (2017).

${ }^{5}$ C. Fang, H. M. Weng, X. Dai and Z. Fang, Chinese Phys. B 25, 117106 (2016).

${ }^{6}$ G. W. Winkler, Q. S. Wu, M. Troyer, P. Krogstrup and A. A. Soluyanov, PRL 117, 076403 (2016).

7 R. Yu, Z. Fang, X. Dai, H. M. Weng, Front. Phys. 12, $127202(2017)$

${ }^{8}$ L. X. Yang, Z. K. Liu and Y. Sun et al., Nat. Phys. 11, 728 (2015).

9 S. M. Huang et al. Nat. Commun. 6, 7373 (2015).

10 S. Y. Xu et al. Science 349, 613 (2015).

11 B. Bradlyn, J. Cano, Z. Wang, M. G. Vergniory, C. Felser, R. J. Cava and B. A. Bernevig, Science 353, aaf5037 (2016).

12 H. Weng, C. Fang, Z. Fang and X. Dai, Phys. Rev. B 93, 241202(R) (2016).

13 H. Weng, C. Fang, Z. Fang and X. Dai, Phys. Rev. B 94, 165201 (2016).

14 Z. M. Zhu, G. W. Winkler, Q. S. Wu, J. Li and A. A. Soluyanov, Phys. Rev. X 6, 031003 (2016).

15 Z. Li, H. Chen, S. Jin, D. Gan, W. Wang, L. Guo, X. Chen, Cryst. Growth Des. 16, 1172 (2016).

16 G. Z. Qin, Z. Z. Qin, H. M. Wang and M. Hu, Phys. Rev. B 95, 195416 (2017).

17 S. D. Guo, J. Phys.: Condens. Matter 29435704 (2017).

18 S. D. Guo, Y. H. Wang and W. L. Lu, New J. Phys. 19 113044 (2017).
19 T. Ouyang, H. P. Xiao, C. Tang, M. Hu and J. X. Zhong, Phys. Chem. Chem. Phys. 18, 16709 (2016).

20 B. Peng, H. Zhang, H. Z. Shao, H. L. Lu, D. W. Zhang, H. Y. Zhua, Nano Energy 30, 225 (2016).

${ }^{21}$ S. D. Guo and B. G. Liu, arXiv:1711.06958 (2017).

${ }^{22}$ G. Kresse, J. Non-Cryst. Solids 193, 222 (1995).

23 G. Kresse and J. Furthmüller, Comput. Mater. Sci. 6, 15 (1996).

24 J. P. Perdew, K. Burke and M. Ernzerhof, Phys. Rev. Lett. 77, 3865 (1996)

25 G. Kresse and D. Joubert, Phys. Rev. B 59, 1758 (1999).

26 A. Togo, L. Chaput and I. Tanaka, Phys. Rev. B 91, 094306 (2015).

27 A. Togo, F. Oba, and I. Tanaka, Phys. Rev. B 78, 134106 (2008).

28 L. Lindsay, D. A. Broido and T. L. Reinecke, Phys. Rev. Lett. 111, 025901 (2013).

29 X. Gu and R. Yang, Appl. Phys. Lett. 105, 131903 (2014).

30 L. Lindsay, D. Broido, and T. Reinecke, Phys. Rev. Lett. 111, 025901 (2013).

31 L. Lindsay, D. Broido, and T. Reinecke, Phys. Rev. B 87, 165201 (2013).

32 Z. Z. Qin, G. Z. Qin, X. Zuo, Z. H. Xiong and M. Hu, Nanoscale 9, 4295 (2017).

33 D. V. Suetin, I. R. Shein and A. L. Ivanovskii, phys. stat. sol. (b) 245, 1590 (2008).

34 H. M. Ledbetter, J. Phys. Chem. Ref. Data 6, 1181 (1977).

35 Z. J. Wu, E. J. Zhao, H. P. Xiang, X. F. Hao, X. J. Liu and J. Meng, Phys. Rev. B 76, 054115 (2007).

36 D. M. Teter, MRS Bull. 23, 22 (1998).

37 S. H. Jhi, J. Ihm, S. G. Louie, and M. L. Cohen, Nature 399, 132 (1999).

38 Z. G. Wu, X. J. Chen, V. V. Struzhkin and R. E. Cohen, Phys. Rev. B 71, 214103 (2005).

39 B. Peng, D. Q. Zhang, H. Zhang, H. Z. Shao, G. Ni, Y. Y. Zhu and H. Y. Zhu, Nanoscale 9, 7397 (2017).

40 A. X. Zhang, J. T. Liu, S. D. Guo and H. C. Li, Phys. Chem. Chem. Phys. 19, 14520 (2017). 